Proyecciones

Vol. 24, No 2, pp. 175-179, August 2005.

Universidad Católica del Norte

Antofagasta - Chile

\title{
ON FOLIATIONS WITH RATIONAL FIRST INTEGRAL
}

\author{
SERGIO LICANIC \\ Universidade Federal Fluminense, Brazil \\ Received: June 2004. Accepted: August 2005
}

\begin{abstract}
We use the Kodaira's classification of relatively minimal elliptic fibrations to prove that a holomorphic foliation in $\mathbf{C P}^{2}$ with a dicritical compact elliptic curve has rational first integral.
\end{abstract}

Keywords : Compact complex surfaces, Holomorphic foliation, Invariant compact curves.

AMS classification : $34 A 20-58 F 18$ 


\section{Introduction and statement of the result}

Given a polynomial system of equations of first order and of first degree in the complex plane Poincar, among others, raised the following question: under which assumptions the system is algebraically integrable. That is, when can one find a rational function such that the solutions of the system are its level curves? (such function is called a rational first integral in modern terminology). On the other hand, admitting the existence of a rational first integral, Poincaré showed how to construct it as soon an upper bound for the degrees of the solutions of the system is given [8], [9], [10] rising the nowadays known Poincar problem (see, for instance, [7] and references there for a short account on this problem).

Interested in Poincar's problem, in [6], Lins Neto exhibits families of foliations in $\mathbf{C P}^{2}$ of degree 4 parameterized by $\mathbf{C}$ such that they all have a singular algebraic solution, specifically the curve formed by the union of a very particular configuration of nine projective lines. Their singularities on that curve are all of radial type while the remained ones have all local holomorphic first integral. Also, for a non-countable dense set of parameter values, these foliations do not have rational first integral at all but for the complement of that set they do have.

Thus, incidentally, Lins Neto's example seems to suggest that some condition on the arrangement of the irreducible components of the algebraic solution -or, rather, on the relative position of the singularities of the foliation- should be given in order to obtain a positive answer to the integrability problem.

Definition 1.1. We say that a singularity of $\mathcal{F}$ is of dicritical radial type if both, $\mathcal{F}$ has algebraic multiplicity one at it, and if after one single blow up the exceptional line is not a solution of the strict transform of $\mathcal{F}$.

Definition 1.2. Let $S$ be an algebraic solution of $\mathcal{F}$, that is $S$ is a reduced divisor such that $S \backslash \operatorname{Sing}(\mathcal{F})$ is a leaf of $\mathcal{F}$. We will say that $S$ is of dicritical radial type if both, any singularity of $\mathcal{F}$ on $S$ is of dicritical radial type and if after one single blow up at each singularity of $\mathcal{F}$ in $S$ the proper transform of $S$ is free of singularities of the proper transform of $\mathcal{F}$.

Our result is the following; 
Theorem Let $\mathcal{F}$ be a holomorphic foliation in $\mathbf{C P}^{2}$ with non-degenerated singularities (i.e. Milnor number one). Let $S$ be a dicritical radial type smooth elliptic curve such that:

a) The singularities of $\mathcal{F}$ in $\mathbf{C P}^{2}-S$ have a finite number of separatrices of $\mathcal{F}$.

b) There exists another compact curve, say $T$, such that $\operatorname{deg}(T)=$ $\operatorname{deg}(S)$ and $S \cap T=\operatorname{Sing}(\mathcal{F}) \cap S$.

Then $\mathcal{F}$ has rational first integral.

\section{Proof of the result}

We use the same notations and definitions for the theory of foliations as appear in [7].

Let $G$ be the pencil generated by $T$ and $S$. Let $\pi: X \rightarrow \mathbf{C P}^{2}$ be the blow up one time each point of the base locus of the pencil $G$ and call $\tilde{G}$ and $\tilde{\mathcal{F}}$ the strict transformed foliation of $G$ and $\mathcal{F}$ by $\pi$ respectively.

We have that $\tilde{G}$ is a fibration over $\mathbf{C} \mathbf{P}^{1}$, that is there exists a proper holomorphic map $g: X \rightarrow \mathbf{C P}^{1}$ such that the leaves of $\tilde{G}$ are exactly the fibers of $g$. Note that the fibers of $g$ are free of exceptional curves. Moreover we assert that the fibers of $g$ are connected. In fact since the singularities of $\mathcal{F}$ in $S$ are admissible and $S$ is smooth we have by CamahoSad index theorem $S^{2}=\#(\operatorname{Sing}(\mathcal{F}) \cap S)$. If $S^{\prime}$ is a curve of the pencil $G$ (by a curve of the pencil we means a curve which is linearly combination of $S$ and $T$ ), we have $\operatorname{deg}\left(S^{\prime}\right)=\operatorname{deg}(S)$ then, by Bezout's theorem, $S^{2}=$ $\sum_{p \in \operatorname{Sing}(\mathcal{F}) \cap S}\left(S, S^{\prime}\right)_{p}$, where $\left(S, S^{\prime}\right)_{p}$ is the intersection number between $S$ and $S^{\prime}$ in $p$. Since $\left(S, S^{\prime}\right)_{p}>0$ for every $p \in \operatorname{Sing}(\mathcal{F}) \cap S$ we have that $\left(S, S^{\prime}\right)_{p}=1$, that is any curve of the pencil $G$ meets $S$ transversally. Then the fibers of $g$ are connected and therefore $\tilde{G}$ is a relatively minimal elliptic fibration over $\mathbf{C P}^{1}$ (a fibration which fibers are connected and free of exceptional curves is called relatively minimal).

We will prove that $\mathcal{F}=G$. First we observe that $\tilde{G}$ has singular fibers. Otherwise, if $\tilde{G}$ has not singular fibers, we would have $c_{1}^{2}(X)=2 c_{2}(X)$ (recall that for any elliptic fibration $f: Z \rightarrow \mathbf{C P}^{1}$ without singular fibers we have the relation $\left.c_{1}^{2}(Z)=2 c_{2}(Z)\right)$ which is an absurd since $c_{1}^{2}(X)=$ $c_{1}^{2}\left(\mathbf{C P}^{2}\right)-9=0$ and $c_{2}(X)=c_{2}\left(\mathbf{C P}^{2}\right)+9=12$.

Let $\tilde{f}$ be a singular fiber of the fibration $\tilde{G}$ and suppose that $\tilde{f}$ is not invariant by $\tilde{\mathcal{F}}$, then by [3], lemma 2 , and lemma $3 \operatorname{tang}(\tilde{\mathcal{F}}, \tilde{f})=c_{1}\left(N_{\tilde{\mathcal{F}}}\right) \tilde{f}-$ $\chi(\tilde{f})=c_{1}\left(N_{\tilde{\mathcal{F}}}\right) \tilde{S}=0$, then $\operatorname{tang}(\tilde{\mathcal{F}}, \tilde{f})=0$ and this implies that $\tilde{f}$ is smooth, contradiction. Therefore $\tilde{f}$ is invariant by $\tilde{\mathcal{F}}$. 
On the other hand the Kodaira table for relatively minimal elliptic fibrations says that any singular fiber of a relatively minimal elliptic fibration is, as a curve, either rational with a cusp or rational with a singularity of nodal type or a chain of -2 curves (that is, a chain of rational smooth curves with self-intersection number -2). Moreover the singularities of a chain of -2 curves are either of nodal type or a singularity formed by the contact of order two between two rational smooth curves or a simple triple crossing between tree rational smooth curves that meet transversally in a point (see [2] pag. 150).

We assert that the singular fibers of $\tilde{G}$ have only nodal type singularities. Indeed if $p$ is a singularity of a chain of -2 curves, then $p$ must be a singularity of $\tilde{\mathcal{F}}$ and therefore must be non-degenerated. Now, if $p$ were a singularity formed by the contact of order two between two rational smooth curves or a simple triple crossing between tree rational smooth curves we would have, by theorem 4 in [4], that the Milnor number of $\mathcal{F}$ in $p$ would be greater than one contradicting the hypothesis that the singularities of $\mathcal{F}$ are non-degenerated. Therefore $p$ must be a nodal type singularity.

By the same reason a fiber of $\tilde{G}$ cannot be rational curve with a cusp. Therefore all singular fibers of $\tilde{G}$ have only nodal type singularities. Thus if $f$ is the projection of $\tilde{\mathcal{F}}$ to $\mathbf{C} \mathbf{P}^{2}$ by $\pi$ we have that $f$ is a $\mathcal{F}$-invariant compact curve of degree 3 with only nodal type singularities which meets $S$ transversally. Observe that $S^{2}=\#(\operatorname{Sing}(\mathcal{F}) \cap S)=G S V(\mathcal{F}, S)=c_{1}\left(N_{\mathcal{F}}\right) S-S^{2}$, that is, $c_{1}\left(N_{\mathcal{F}}\right) S=2 S^{2}$. Therefore, by [5] theorem 1, we obtain that $\mathcal{F}$ is given in homogeneous coordinates in $\mathbf{C}^{3}$ by the meromorphic 1-form $\omega=d h / h-d g / g$ where $h$ and $g$ are homogeneous polynomials in $\mathbf{C}^{\mathbf{3}}$ of the same degree and such that $S=\{h=0\}$ and $f=\{g=0\}$. That is $\mathcal{F}$ has rational first integral $h / g$. Consequently $G=\mathcal{F}$.

\section{References}

[1] P. Baum and R. Bott, On the zeroes of meromorphic foliations, Essais en l'honeur de G. De Rham, pp. 29-47, (1970).

[2] W. Barth, C. Peters, A. Van de Ven, Compact complex surfaces, Springer Ergebnisse 4, (1984).

[3] M. Brunella,Feuilletages holomorphes sur les surfaces complexes compactes, Ann. Sci. E. N. S. 4, Vol. 30, pp. 569-594, (1997). 
[4] C. Camacho, A. Lins Neto and P. Sad, Topological invariants and equidesingularization for holomorphic vector field, Jour. Diff. Geom. vol. 20, pp. 143-174, (1984).

[5] D. Cerveau and A. Lins Neto, Holomorphic foliation in $\mathbf{C} P^{2}$ having an invariant algebraic curve, Ann. Inst. Fourier, Grenoble 41, 4, pp. 883-903, (1991).

[6] A. Lins Neto Some examples for the Poincaré and Painlevé problems, Ann. Sci. École Norm. Sup. (4) 35, No. 2, pp. 231-266, (2002).

[7] S. Licanic, An upper bound for the total sum of the Baum-Bott indexes of a holomorphic foliation and the Poincare Problem, Hokkaido Mathematical Journal Vol. 33, No.3, October, (2004).

[8] H. Poincaré Sur líntégration algébrique des équations différentielles, C. R. Acad. Sci. 112, pp. 761-764, (1891).

[9] H. Poincaré Sur l'intégration algébrique des équations différentielles du premier ordre et du premier degré, Rend. Circ. Mat. Palermo 5, pp. 161-191, (1891).

[10] H. Poincaré Sur l'intégration algébrique des équations différentielles du premier ordre et du premier degré , Rend. Circ. Mat. Palermo 11, pp. 193-239, (1897).

\section{Sergio Licanic}

Dep. de Matemática Aplicada

Universidade Federal Fluminense UFF

Rua Mario Santos Braga s/n

Praça do Valonginho-Centro-Niteroi

CEP: 24020-140

Brazil

e-mail : ser@impa.br 